\title{
Recall of lost-to-follow-up pre-antiretroviral therapy patients in the Eastern Cape: Effect of mentoring on patient care
}

\author{
Martin Jones, Maryna Stander, Marlene van Zyl, David Cameron
}

Background. In 2011 an experienced HIV nurse from the UK was deployed for 3 months to act as a mentor to nurses learning to initiate antiretroviral therapy (ART) in primary care clinics in a small town in the Eastern Cape, South Africa.

Methods. A review of existing pre-ART patient files $(N=286)$ was carried out and lost-to-follow-up (LTFU) HIV patients were recalled.

Results. Only $24 \%$ of patients had attended the clinics within the preceding 6 months and 20\% had not attended for longer than 2 years. Two lay counsellors visited 222 patients to encourage them to return to care; $65 / 286$ (23\%) were untraceable, $11 / 286(4 \%)$ had relocated, 30/286 (10\%) declined, and 8/286 (3\%) had died. In the 6 weeks following recall, $51 / 286$ patients (18\%) returned to the clinics.
CD4 count testing was repeated and screening for tuberculosis (TB) and other opportunistic infections was performed for all patients; ART was initiated in 13/51 (25\%), 1 patient tested positive for TB, and isionazid (INH) prophylaxis was initiated in 23/51 (45\%). The cost of recall was R130/patient. Within 6 months, all clinics began providing full ART services, 17 professional nurses were mentored and they initiated ART in 55 patients.

Conclusions. Mentoring plays an important role in professional nurse training and support. Recall of LTFU patients is feasible and effective in improving ART services in rural settings.

S Afr Med J 2012;102(9):768-769. DOI:10.7196/SAMJ.5957
In September 2011 a nurse with 25 years' experience in HIV clinics in the UK was dispatched by the Global Medic Force and deployed by the Foundation for Professional Development (FPD) for a 3-month volunteer mentorship in the central Karoo town of Graaff-Reinet, Cacadu District, Eastern Cape, South Africa (SA). In 2009 an HIV prevalence of $24.3 \%$ was reported for women tested during antenatal visits in the Cacadu district. ${ }^{1}$

The mentorship assignment was to support primary care nurses trained in nurse initiation and maintenance of antiretroviral therapy (NIMART). The urgent need to expand ART access to $80 \%$ of eligible HIV-positive people has made it essential that nurses working in SA primary healthcare clinics (PHCs) share the task of initiating ART and subsequent patient management. ${ }^{2,3}$ In addition to training, nurses require regular support and mentoring to perform this task competently., ${ }^{4,5}$

Graaff-Reinet has 3 community clinics providing primary healthcare to 60000 inhabitants. ${ }^{6}$ The surrounding Camdeboo subdistrict is served by 2 community clinics and a mobile PHC service. Each clinic has 2 professional nurses and receives a weekly visit from a doctor. Prior to 2011, all patients diagnosed with HIV and in need of ART were referred to specialist HIV clinics at the local hospital and the Graaff-Reinet Community Health Centre. Follow-up of patients not yet eligible for ART was performed at local clinics. All primary care nurses attended NIMART training prior to September 2011,

East Sussex Healthcare NHS Trust, Eastbourne, United Kingdom Martin Jones, RN, MSc

Foundation for Professional Development, Eastern Cape Maryna Stander, RN

Marlene van Zyl, RN

Department of Family Medicine, University of Pretoria, and Foundation for Professional Development, Pretoria

David Cameron, MB ChB, M Prax Med, MPhil, FCFP (SA) but lacked the supervision and mentoring to enable them to begin initiating ART.

Loss to follow-up among HIV patients prior to ART eligibility is reportedly very high. Only $18 \%$ of patients in sub-Saharan Africa who are not yet eligible for ART at HIV diagnosis remain continuously in care until eligibility. ${ }^{7}$ Larson ${ }^{8}$ found that $74 \%$ of patients with a CD4 count $>350$ and $59 \%$ with a CD4 count of 251 - 350 failed to return to a public clinic in Johannesburg within 1 year.

Loss to follow-up is associated with significant mortality. Of lostto-follow-up (LTFU) pre-ART patients in Jinja, Uganda, 28\% died within 1 year of last attendance. ${ }^{9}$ A $21 \%$ mortality rate was identified among LTFU patients from a large clinic in Johannesburg. ${ }^{10}$

An efficient way to identify and attend to service gaps is to investigate LTFU patients. ${ }^{11}$ To assess existing HIV care in PHCs in Graaff-Reinet, the case notes of all patients diagnosed with HIV between 2005 and 2011 were reviewed. We report the findings and outcomes of a subsequent recall exercise.

\section{Methods}

All pre-ART patient case notes in the 3 PHCs in Graaff-Reinet were reviewed. Each patient's name, address, date of last attendance and last recorded CD4 count was recorded. Names were cross-referenced against the databases of the specialised HIV clinics to determine if they were receiving ART elsewhere. Two lay counsellors were deployed to visit the remaining patients to invite them to re-attend the clinic for review.

\section{Results}

Only 60 (21\%) patients attended a PHC and had their CD4 count determined in the preceding 6 months. Five files without HIVrelated data were excluded from analysis. Most files contained limited data - typically only consent for HIV testing and laboratory reports. All pre-ART registers were incomplete and there were no systems for ensuring receipt of regular HIV care, including national recommendations of 6-monthly monitoring of CD4 count.

Within 6 weeks, 221 patients identified for recall were contacted (Table 1). Most were grateful for the follow-up reminder; a few requested confirmatory HIV-testing (all were positive); and 30 (10\%) declined to re-attend, either due to prior attendance within the 
Table 1. Outcome of recall of LTFU pre-ART patients from 3 primary care clinics $(N=286)$

\begin{tabular}{ll}
\hline & $\boldsymbol{n}(\%)$ \\
\hline Already attending specialised HIV clinics & $121(42)$ \\
Relocated & $11(4)$ \\
Died & $8(3)$ \\
Declined to attend & $30(10)$ \\
Not traced & $65(23)$ \\
Attended for review & $51(18)$
\end{tabular}

preceding 6 months or unwillingness to disclose their HIV status to their families. Eight (3\%) patients had died, 11 (4\%) relocated and 65 (23\%) could not be traced. Fifty-one patients re-attended for review within the 6-week period.

CD4 count testing and tuberculosis (TB)/opportunistic infection screening was performed for all patients; 1 was found to have active TB and the appropriate treatment was initiated; 13 (25\%) were fasttracked to start ART; and 23 patients accepted the offer to receive isoniazid (INH) prophylactic therapy (IPT) (Table 2). Cotrimoxazole was provided to all patients with a CD4 count $<200$ and women were advised to have a Papanicolaou (Pap) smear. Furthermore, all patients were encouraged to have their children tested. The pre-ART, ART and IPT registers were updated, the filing system was improved, and the comprehensive Adult Care Record was completed for each patient.

\section{Discussion}

The ethical issue of deploying lay counsellors to recall patients was comprehensively discussed at the project start. Although from the same community as the patients, their work involved counselling and HIV-testing, and all counsellors signed a confidentiality agreement on assuming their role. Patient rights to confidentiality and autonomy were respected and acknowledged. In the light of preventable immunodeficiency, the risk of opportunistic infections in unmonitored patients, and onward HIV transmission, recall was considered to be in the best interests of the patients. Patients retained the right to decline re-attendance. Refusal in some demonstrated the need to increase efforts to reduce stigma and improve patient-centred service. ${ }^{12}$

Effective mentoring balances 3 key elements - support, challenge and a vision for the future ${ }^{13}$ - all of which contributed to the success of this project. Good relationships were initially established, current practice was assessed and gaps in the standard of care were identified. A clear vision of how an effective ART service should run was explained. The entire team was mobilised to act and within 6 weeks, 51 HIV patients returned to follow-up and care. Concurrently, systems and tools were put in place to maintain effective patient management.

A challenge of any quality improvement strategy is sustainability. Evidence of sustained improvement was seen 6 months after the initial project: all 5 PHCs in the Camdeboo sub-district had started providing full ART services; 17 professional nurses had been mentored and had

Table 2. Outcomes of pre-ART patient reviews $(N=51)$

\begin{tabular}{ll}
\hline & $\boldsymbol{n}(\%)$ \\
\hline ART initiated immediately & $13(25)$ \\
IPT initiated & $23(45)$ \\
TB treatment initiated & $1(2)$ \\
Pre-ART follow-up & $24(47)$
\end{tabular}

initiated ART in 55 patients; the dispensaries of all 5 clinics were fully stocked with ART drugs; patient records and registers were completed properly; and all eligible patients were receiving IPT.

Loss to follow-up indicates a problem in ART service delivery. ${ }^{11}$ Tracing LFTU patients can be costly and of limited success. Rosen and Ketlhapile ${ }^{10}$ could telephonically trace $260 / 495$ patients who were 1 month late for their ART appointments; 55 (21\%) had died, 135 (51\%) were still attending treatment at the same or another site and 70 (27\%) discontinued ART; only 20 (4\%) returned to care at a cost of R3 280/patient. In this project, by making use of lay counsellors to perform home visits, it was possible to recall $51(18 \%)$ patients at a cost of R130/patient.

\section{Conclusions}

Sharing the responsibility of initiating ART with primary care nurses throughout SA is ambitious. Mentoring plays an important role in training, support and clinical skills development. PHC systems must be accurate and robust to enable HIV patients to receive ongoing comprehensive care guided by tools such as the Adult Care Record and ART registers. In this work, an extended visit by an experienced international mentor contributed to raising HIV care standards in PHCs in a rural area.

Regular file and register review and timely recall can ensure that HIV patients receive continuous care, and that preventable morbidity and mortality are indeed prevented. The use of lay counsellors familiar with the community can be an effective means of reaching LTFU patients. LFTU patient recall is feasible and cost-effective, even in resource-constrained situations.

Acknowledgements. We acknowledge the Global Medic Force - a development agency providing clinical expertise to train local workers in emerging nations (http://www.globalmedicforce.org). Volunteer mentor travel and accommodation costs were provided by the President's Emergency Plan for AIDS Relief (PEPFAR), through the Foundation for Professional Development (FPD). Ethical approval was obtained from the FPD Research Ethics Committee.

\section{References}

1. Department of Health. National Antenatal Sentinel HIV and Syphilis Prevalence Survey in South Africa. Pretoria: DoH, 2009. http://www.health-e.org.za/documents/85d3dad6136e8ca9d02cceb7f Africa. Pretoria: DoH, 2009. http

2. Department of Health. National Strategic Plan on HIV, STI and TB 2012 - 2016. Pretoria: DoH, 2012 Department of Health. National Strategic Plan on HIV, STI and TB 2012 - 2016.
http://www.doh.gov.za/docs/stratdocs/2012/NSPfull.pdf (accessed 8 April 2012).

3. Walensky R, Wood R, Weinstein MC, et al. Scaling up antiretroviral therapy in South Africa: the impact of speed on survival. J Infect Dis 2008;197:1-9. [http://dx.doi.org/10.1086/587184]

4. Department of Health. Clinical Mentorship Manual for Integrated Services. Pretoria: DoH, 2011 http://www.doh.gov.za/docs/policy/2011/clinicalmentorship.pdf (accessed 8 April 2012)

5. World Health Organization. Recommendation for Clinical Mentoring to support Scale-Up of HIV Care, Antiretroviral Therapy and Prevention in Resource-Constrained Settings. Geneva: WHO, 2006 http://www.who.int/hiv/pub/guidelines/clinicalmentoring.pdf (accessed 8 April 2012).

6. Population Of. Population of Graaff Reinet. http://population-of.com/en/South-Africa/05/Graaff Reinet/ (accessed 8 April 2012)

Rosen S, Fox M. Retention in HIV care between testing and treatment in sub-Saharan Africa: A systematic review. PLoS Medicine 2011;8(7):e1001056. [http://dx.doi.org/10.1371/journal.pmed.1001056]

8. Larson B, Brennan A, McNamara L, et al. Early loss to follow-up after enrolment in pre-ART care at Larson B, Brennan A, McNamara L, et al. Early loss to follow-up after enrolment in pre-ART care at a
large public clinic in Johannesburg, South Africa. Top Med Int Health 2010;15(1):43-47. [http://dx.doi. large public clinic in Johannesburg, Sout

9. Amuron B, Namara G, Birungi J, et al. Mortality and loss to follow-up during the pre-treatment period in an antiretroviral therapy programme under normal service conditions in Uganda. BMC Public Health 2009;9:290. [http://dx.doi.org/10.1186/1471-2458-9-290]

10. Rosen S, Kethapile M. Cost of using a patient tracer to reduce loss to follow-up and ascertain patien status in a large antiretroviral therapy program in Johannesburg, South Africa. Top Med Int Health 2010;15(1):98-104. [http://dx.doi.org/10.1111/j.1365-3156.2010.02512.x]

11. Geng EH, Glidden DV, Emenyonu N, et al. Tracking a sample of patients lost to follow-up has a majo impact on understanding determinants of survival in HIV-infected patients on antiretroviral therapy in Africa. Top Med Int Health 2010;15(1):63-69. [http://dx.doi.org/10.1111/j.1365-3156.2010.02507.x]

12. McGuire M, Munyenyembe T, Szumilin E, et al. Vital status of pre-ART and ART patients defaulting from care in rural Malawi. Top Med Int Health 2010;15(S1):55-62. [http://dx.doi.org/10.1111/j.13653156.2010.02504.x]

13. Bower DJ, Diehr S, Morzinski JA, Simpson DE. Support-challenge-vision: a model for faculty mentoring. Medical Teacher 1998;26(6):595-597. 